\title{
Implementation of K-Means Algorithm and Dynamic Routing Protocol in VANET
}

\author{
Manoj Sindhwani ${ }^{1}$, Charanjeet Singh ${ }^{1, *}$ and Rajeshwar Singh ${ }^{2}$ \\ ${ }^{1}$ School of Electronics and Electrical Engineering, Lovely Professional University, Phagwara, 144411, India \\ ${ }^{2}$ Doaba Group of Colleges, Nawanshar, 144517, India \\ ${ }^{*}$ Corresponding Author: Charanjeet Singh. Email: rcharanjeet@gmail.com \\ Received: 10 March 2021; Accepted: 01 May 2021
}

\begin{abstract}
With the growth of Vehicular Ad-hoc Networks, many services delivery is gaining more attention from the intelligent transportation system. However, mobility characteristics of vehicular networks cause frequent disconnection of routes, especially during the delivery of data. In both developed and developing countries, a lot of time is consumed due to traffic congestion. This has significant negative consequences, including driver stress due to increased time demand, decreased productivity for various personalized and commercial vehicles, and increased emissions of hazardous gases especially air polluting gases are impacting public health in highly populated areas. Clustering is one of the most powerful strategies for achieving a consistent topological structure. Two algorithms are presented in this research work. First, a k-means clustering algorithm in which dynamic grouping by k-implies is performed that fits well with Vehicular network's dynamic topology characteristics. The suggested clustering reduces overhead and traffic management. Second, for inter and intra-clustering routing, the dynamic routing protocol is proposed, which increases the overall Packet Delivery Ratio and decreases the End-to-End latency. Relative to the cluster-based approach, the proposed protocol achieves improved efficiency in terms of Throughput, Packet Delivery Ratio, and End-to-End delay parameters comparing the situations by taking different number of vehicular nodes in the network.
\end{abstract}

Keywords: Cluster head; cluster member; intelligent transportation system; packet delivery ratio; vehicular communication

\section{Introduction}

The Vehicular Ad hoc Networks (VANETs) are research network that pursues the future of pervasive computing [1]. VANETs are one of the Intelligent Transportation System (ITS) impact zones that allow vehicle drivers to interact and synchronize with each other to prevent dangerous conditions before they happen, thus enhancing driver protection and relaxation [2]. Inter-Vehicle Interaction is required to understand operational route planning, traffic situation control, emergency-message transmission and secure driving [3]. Generally, VANETs applications are time-crucial. The main constraints of these applications are the rapid propagation of data across the network area in question. Approximately $60 \%$ of

This work is licensed under a Creative Commons Attribution 4.0 International License, which permits unrestricted use, distribution, and reproduction in any medium, provided the original work is properly cited. 
road accidents can be avoided by the prompt distribution of emergency signals to local and remote vehicles. The propagation of broadcast messages is distributed into two types: single-hop and multi-hop transmission, as shown in Fig. 1. In a flat V2V dense network, a conventional multi-hop broadcast message propagation scheme will result in packet drop, high communication costs, high data packets delivery delay. We need a stable communication infrastructure for message propagation to address the disadvantages mentioned earlier of a flat V2V network system. VANETs cluster-based networking system forms a trusted backbone for the efficient transmission of messages and associated reserves all vehicles inside the network [4,5]. The application of VANETs, such as security and complex road situation details, needs the extremely static topology of the network, so the enhanced clustering technique is highly required in such instances. The clustering shows an essential way of shaping community vehicles and efficiently coordinating wireless communications.

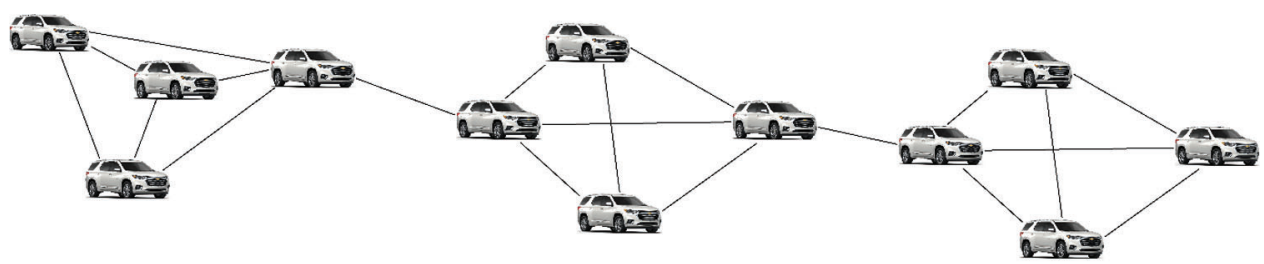

(a)

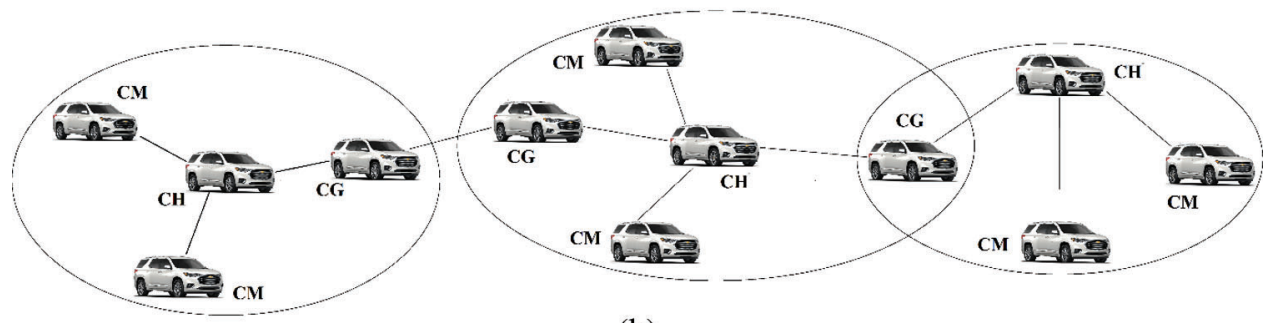

(b)

Figure 1: Flat and cluster-based structure in VANET a) Flat network structure b) Cluster based network structure

In VANETs, clustering routing contributes to decreased network dynamics [6]. Due to various formation requirements, clustering mechanisms often vary from each other. According to functionality and its application domain, these requirements may differ. Nodes can, however, serve as cluster members (CMs) in the cluster or can be selected as cluster head $(\mathrm{CH})$.

CMs are normal nodes, whereas CHs perform transmission of information in VANETs [7] between clusters and intra-clusters, as indicated in Fig. 2. CHs are then chosen to achieve optimized network efficiency based on their improved functionality. In order to achieve efficient communication, $\mathrm{CH}$ selection is therefore necessary. Therefore, we propose a dynamic clustering protocol utilizing k-means for $\mathrm{CH}$ selection and cluster forming in this paper, which increases the overall distribution ratio of packets and reduces VANETs end-to-end latency. The clustering of K-means divides the region into four segments, and each segment has several CHs.

The objective function is then determined using key vehicle parameters, such as position $(\mathrm{X}, \mathrm{Y})$, speed, direction and point of interest (POI). The measured objective function value helps create more stable clusters and takes advantage of the data transfer process by choosing more stable routes. Each cluster has particular interests through this clustering, such as parking data, accident alerts, and overcrowding information. When a $\mathrm{CH}$ receives a message, it tests whether or not the vehicles within the cluster are involved in the message. If they are interested in the vehicles within the cluster, $\mathrm{CH}$ will 
transmit the data to its members. Else, would transmit the data to the next $\mathrm{CH}$. This will decrease the nonrelevant distribution of data in the network. The data is transmitted via the nearest neighboring $\mathrm{CHs}$ in this path, which is established from the vehicles' position.

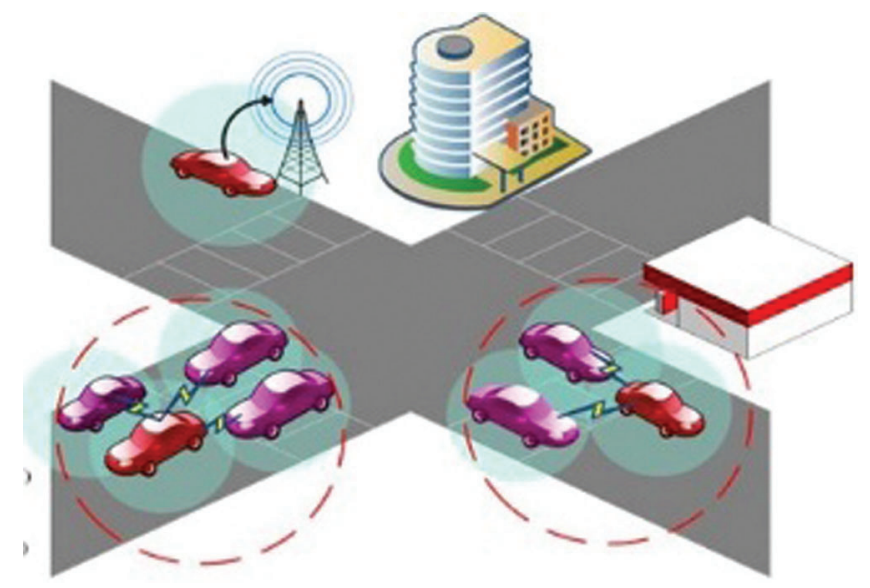

Figure 2: Node clustering in VANETs

The main objective of this paper is to create a clustering protocol that increases packet delivery ratio (PDR) .and decreases the end-to-end (E2E) delay via K-means clustering and dynamic routing. First, a clustering algorithm is designed that divides the region into segments by using K-means and then elects CHs takes into account: vehicle position, vehicle direction, vehicle speed, vehicle POI and destination. Each cluster has particular interests through this context-based clustering, such as parking data, accident alerts, and congestion information. Second, a dynamic routing protocol is defined for successful intercluster and intra-cluster transmission.

The portion of the article is arranged as: Section 1 produced the introduction. A literature review is given in Section 2. Section 3 discusses the clustering model. Section 4 implements the dynamic routing protocol. Finally, simulation analysis and conclusions are discussed in Sections 5 and 6, respectively.

\section{Literature Review}

There are numerous clustering procedures and techniques for VANETs in the last many years. In writing [8,9], a few stable clustering-based papers were suggested in VANETs. However, in all these literatures, it is concluded that they do not sustain the quality of the group head due to high vehicle growth and the constantly varying topology of vehicles.

In Bello-Salau et al. [10], a new routing-based algorithm is proposed to improve various essential parameters such as path loss, transmit power, and received signal strength. This algorithm also improves the reliability of the network. Their findings suggest that the algorithm improves road anomaly vehicle communication, thus intimating drivers to navigate anomalous roads to reduce road accidents.

In Song et al. [11], a cluster-based directional routing (DBR) protocol is suggested where a node sends data to a nearby $\mathrm{CH}$ whose moving path is identical to the message's communication direction. The communication path is determined by a node's location and destination location coordinates. The authors suggested an enhanced greedy traffic-aware routing protocol (GyTAR) in Jerbi et al. [12] that is a spatial routing protocol centered on an intersection. It uses the idea of clusters among adjacent intersections to transmit the data. 
The routing protocol for VANETs for vehicle intensity and load-aware (VDLA) was suggested in Zhao et al. [13], which chooses a set of junctions to create the path to the destination. The option is established on the vehicle's density in real-time, the traffic density and the distance to the target.

A cluster-based VANET connectivity maintenance algorithm called AODV-CV is suggested in Abuashour et al. [14], where the $\mathrm{CH}$ is chosen based on all vehicles' velocity within the cluster [15] region. By increasing the velocity, the AODV-CV performs better than AODV in terms of throughput. In Louazani et al. [16], a new Cluster Based Routing (CBR) protocol named CBVANET was suggested. The architecture of the clustering system for communication between VANET vehicles was the focus of this model. By decreasing the cluster formation duration, election duration and shifting duration, this model reduced the delay in VANET. The least velocity vehicle was selected as $\mathrm{CH}$. The AODV-CV performs better in generation time and shifting time.

In Malathi et al. [17], the authors presented a cluster-based routing protocol that takes into account the target of a vehicle and the perspective for $\mathrm{CH}$ selection and routing. However, the proposed work is based on dynamic clustering that maximizes the clustering messages overhead.

A new algorithm for the system to sort a cluster architecture and $\mathrm{CH}$ election suitable for vehicular networks is suggested in Mohammed Nasr et al. [18]. Moreover, it shows a novel clustering-based routing approach that ensures efficient data transmission among the vehicles.

A new protocol for cluster-based lifetime routing (CBLTR) had suggested in Abuashour et al. [14]. This protocol aims to maximize the stability of the route and average throughput, reduce the E2E delay and decrease clustering overhead messages.

\section{Clustering in Vehicular Ad-hoc Network}

K-means is one of the most effective data extracting procedures $[19,20]$ among clustering algorithms, mainly because of its simplicity, scalability and because it is easy to adjust to different scenarios and domains. There are some well-known shortcomings in k-means, however. To be exact, the number of clusters, $\mathrm{k}$, is required as an input. For the request for data clustering, Influential $\mathrm{k}$ is answered. Input is given as the number of clusters, and then a modified k-means is employed to split the vehicles into clusters.

For cluster formation, we apply k-means [21] such that cluster creation occurs based on three parameters: the dimension of $\mathrm{x}$, second is the dimension of $\mathrm{y}$, and last is the distance of Euclidean. The central K-means algorithm definition is as follows. First, the road is split into sectors, and the number of clusters for each sector/segment is $\mathrm{k}$. And $\mathrm{K}$ centers for clustering are initialized. The distance from the cluster center is then determined, and the information is split into the closest cluster center. The cluster center is modified based on the outcome of the partition. Until the predefined iterations are attained, this method continues to loop. The final outcomes are acquired at the end of the iteration. The process of $\mathrm{CH}$ selection is weight-based, which considers subsequent factors: location, direction, velocity, and POI. If a vehicle joins any cluster at any unit of time, it becomes a member of the associated cluster and transmits a CH-REQ to the corresponding $\mathrm{CH}$. Each cluster has a threshold level (TL), and it begins the procedure of new $\mathrm{CH}$ election when a $\mathrm{CH}[22,23]$ extends the TL.

The handover procedure is started after $\mathrm{CH}$ selection. Each vehicle must be allocated to a single cluster based on its position for each unit of time. Vehicles are characterized to create clusters based on the following parameters in weight-based clustering:

a) Location: As a significant parameter, the vehicle's location is considered and can be calculated using GPS. This GPS system supplies OBU with information that decides its current position.

b) Direction: A vehicle's path is calculated by measuring the difference among the last two places obtained by the GPS system. 
c) Velocity: The speed of the vehicle is measured by OBU. There should be the least difference in velocity for vehicle nodes present in the same cluster.

d) Point-of-Interest list: There are certain interests in any car. Some vehicles are concerned with data about parking, restaurants nearby, and some are only concerned with information about incidents and overcrowding, etc. A vector is used to describe a vehicle's interests. Each " $k$ " vehicle maintains an interest vector in the way of:

$P I(K)_{I}=\left(P I(K)_{1},\left(P I(K)_{2}, \cdots \cdots\left(P I(K)_{n}\right)\right.\right.$

$P$ In is order for vehicles present in the same cluster, the vehicles' POI should be the same.

The clusters are formed based on the above parameters, and each cluster has one $\mathrm{CH}$. The $\mathrm{CH}$ can sustain a vehicle's interests. When a $\mathrm{CH}$ accepts data, it first verifies whether the cars within the cluster are involved. If there is an interest in the vehicles inside the cluster, $\mathrm{CH}$ will transmit the packet to its members. Else, the message would be routed to the next $\mathrm{CH}$. This will decrease the network communication messages inside the cluster. The process of selecting the cluster head is as follows:

- A weight-based $\mathrm{CH}$ selection algorithm is suggested for optimal $\mathrm{CH}$ selection. Each node calculates a weight according to specific parameters, and the highest weight node is selected as the $\mathrm{CH}$. The total duration required for the $\mathrm{CH}$ collection to be finished is $T$. This is split into four sub-hours. The following measures are involved in $\mathrm{CH}$ selection:

- Each vehicle acquires its clustering factors from its on-board component: position, path, velocity, and POI and the time required for this is $\mathrm{T} 1$.

- After finding its nearby vehicles, each vehicle recognizes its neighbouring vehicles whose POI is similar; each vehicle transmits its clustering factors to its nearby nodes.

- If a node obtains the clustering factors from its neighbours, a list for each nearby is preserved. $\left(N_{\text {List }}\right)$. The time undertook to achieve this is $\mathrm{T} 2$.

- A list comprises the ID of the neighbouring car, its location, speed, destination, POI and compatibility with the POI.

$N_{\text {List }}=\left(N_{1}, N_{2}, \ldots N_{n}\right)$

Three parameters, which are cosine similarity and soft cosine similarity, are used to compute Point-ofinterest compatibility (PC). For instance, the PC is calculated using the following equation between vehicle "a" and vehicle "b" with " $n$ " as the number of neighbouring nodes:

$P C_{a b}=\frac{\sum_{k}^{n} p a_{k} p a_{k}}{\sqrt{\sum_{k=1}^{n} p a_{k}^{2} \sum_{k=1}^{n} p b_{k}^{2}}}+\frac{\sum_{i, j}^{n} s_{i j} a_{i} b_{j}}{\sqrt{\sum_{i, j}^{n} s_{i j} a_{i} b_{j}} \sqrt{\sum_{i, j}^{n} s_{i j} a_{i} b_{j}}}$

cosine similarity soft cosine similarity

Here $s_{i, j}=$ similarity (feature $_{\mathbf{i}}$, feature ) $_{\mathbf{j}}$.

- Next, the mean Euclidean distance (AED) between the "a" vehicle and each of its "b" neighbours is measured using the following equation:

$A U D_{a, b}=\frac{\sum_{k=1}^{n} \sqrt{\left(x_{a_{k}}-x_{b_{k}}\right)^{2}+\left(y_{a_{k}}-y_{b_{k}}\right)^{2}}}{n}$

Here $\left(x_{a}, x_{b}\right)\left(y_{a}, y_{b}\right)$ signifies position coordinates of nodes " $\mathrm{a}$ " and " $\mathrm{b}$ ", and " $\mathrm{n}$ " represents the number of neighbours. The time duration for this process is T3. T4 is static and it is identical for all vehicles nodes. 
- Each vehicle determines the waiting time "Tw"

$T w=\frac{1}{\alpha\left|N_{\text {List }}(k)\right|} \times T 4 \times R$

Here, $\alpha$ signifies the number of times the vehicle " $\mathrm{k}$ " selects as a $\mathrm{CH}$ earlier. $\left|N_{\text {List }}(k)\right|$ is the nearby nodes of node " $\mathrm{k}$ ". $\mathrm{R}$ represents a random number among 0.1 and 0.2 . The node awaits for "Tw" and determines the Weight Value (WV). If any CH request is accepted with in this "Tw", the vehicle does not compute the WV. It agrees that vehicle as a $\mathrm{CH}$.

- Each node computes the WV after "Tw". The node sends out the $\mathrm{CH}$ advertising message immediately after measuring WV. Because of R., each node has different waiting times. The WV is determined using the equation below:

$W V_{k}=\frac{A P C_{k}}{A E D_{k} \cdot A V_{k}}$

In order to enhance WV of a vehicle, $A E D_{k}$ and $A V_{k}$ should be lowest.

\section{Dynamic Routing Protocol}

If the network clustering structure has been created, when the cluster member demands that the packets be transferred to the designated destination [24,25], the packet will be sent to the $\mathrm{CH}$. By using the dynamic routing protocol to the destination, the $\mathrm{CH}$ forwards the packet. Two sub-protocols are divided into the routing protocol.

1. Intra-sector routing protocol

2. Inter-sector routing protocol

\subsection{Intra-sector Protocol}

The suggested routing protocol intends to disseminate the data packets via the chosen $\mathrm{CHs}$ within the section. Each $\mathrm{CH}$ constructs its routing table and saves the neighboring $\mathrm{CH}$ ID and its related places to maintain routing data. When the nearby $\mathrm{CH}$ gets data, it chooses the candidate $\mathrm{CHs}$ situated near the destination regardless of the location of the $\mathrm{CH}$ in its routing table. After that, it sends the data to the nearest $\mathrm{CH}$. If there is no nearby $\mathrm{CHs}$ to the destination node, the local $\mathrm{CH}$ uses a store-and-forward procedure as a recovery procedure; It saves the data in a particular buffer and keeps going until another $\mathrm{CH}$ relay is located. Algorithm 1 describes the steps taken to propagate the data inside the sector. If a node receives data at any point during the simulation, it first verifies its routing table and chooses the $\mathrm{CHs}$ with the least distance to the destination. Lastly, if the routing table is empty, then a store-andforward method follows the current $\mathrm{CH}$.

Proposed Algorithm: Intra-Sector Routing Protocol

Input Parameters:

a) Set of Vehicle nodes, and $\mathrm{CHs}$

b) Distance of each vehicle $\mathrm{CH}$

c) direction of each Vehicle $\mathrm{CH}$

\section{Output Parameters:}

a) Best next forwarding node 


\section{Proposed Algorithm (continued).}

For Each data packet obtained by the $\mathrm{CH}$

If data obtained by $\mathrm{CH}$, then

Check forwarding table of corresponding $\mathrm{CH}$

If forwarding table of $\mathrm{CH}$ Not empty, then

Save the $\mathrm{CHs}$ which are closest to the target in the candidate $\mathrm{CH}$ table

Next forwarding node $=\mathrm{CH}$ near to destination

\section{End If}

\section{Else}

\section{Store and forward}

\section{End If}

\section{End For}

\subsection{Inter-sector Protocol}

The protocol proposed seeks to disseminate the packets across the sector via the selected CHs as described as follows:

- If a source vehicle "s" wishes to transmit data to the vehicle "d", the "s" sends the message including the target location $\left(\operatorname{Tloc}\left(x_{t l}, y_{t l}\right), x y\right)$ to its corresponding $\mathrm{CH}$ " $\mathrm{k}$ ".

- After that, the direction of communication (DC) is computed. DC is related to the path of $\mathrm{CH}$ "c" if their cosine similarity (CSM) is more than 0 . The connection between DC and the velocity Vector $\left(V_{c}\right)$ is calculated utilizing the following equation.

$C S M=\frac{D C V_{c}}{|D C|\left|V_{c}\right|}$

where DC is the distance between the vehicle and the target position is DC. There is a velocity vector in each $\mathrm{CH}$ " $\mathrm{c}$ " that can be defined as

$V_{c}=V_{c} \cdot i+V_{c} \cdot j$

Also, every $\mathrm{CH}$ " $\mathrm{c}$ " has a certain target $\left(x_{\text {dest }}, y_{\text {dest }}\right)$. For selecting the forward node, the distance between the target position and CH's target is also carried.

- To choose the next forwarding $\mathrm{CH}$ node, a $\mathrm{CH}^{\prime}$ c' utilizes the targets and directions of its nearby nodes, $\mathrm{CH}$. Initially, it determines a DC post's communication route. Then, it tests CSM by using Eq. (6) $\mathrm{CH}$ node 'c' for every neighbour, with velocity.

- Subsequently evaluating CSM and $\Delta D_{c}$ for every nearby $\mathrm{CH}$ "c", a $\mathrm{CH}$ " $\mathrm{k}$ " defines the routing metric (RM) for each neighbour $\mathrm{CH}$ "c":

$R M=\frac{C S M}{\Delta D_{c}}$ 
- A neighbour $\mathrm{CH}$ in another sector whose $\mathrm{RM}$ is highest is chosen as the next hop $\mathrm{CH}$.

- Then, the next $\mathrm{CH}$ tests whether Tloc within the cluster is located or not. The message is forwarded to Tloc if Tloc is located within the cluster. Otherwise, the next-hop $\mathrm{CH}$ is selected again using $\mathrm{RM}$, and the process repeats itself.

\subsection{Operation of Proposed Routing Protocol}

- It sends the message to its $\mathrm{CH}$ when a source "S" receives a message.

- The $\mathrm{CH}$ first tests whether or not the Tloc is located inside the cluster.

- If yes, the message is sent to Tloc.

- Otherwise, it will verify that the Tloc is in the segment

i) If so, the intra-segment packet forwarding protocol is utilized, where the next $\mathrm{CH}$ node is selected from its forwarding table.

ii) If Not, the inter-segment protocol is utilized where the next $\mathrm{CH}$ node cantered on the $\mathrm{RM}$ is chosen in the other sector. The next $\mathrm{CH}$ tests Tloc's accessibility within its cluster again. If Tloc is not identified, the next hop $\mathrm{CH}$ is chosen again, and the procedure repeats until the data reaches its Tloc node.

\section{Performance Evaluation}

Two types of simulators are used to assess our proposed protocol's execution: the traffic simulator that replicates the vehicle mobility and the network simulator that creates the vehicular area. The SUMO is the most used traffic simulator in VANET.

A $1000 \times 1000$ area segment is applied to test the proposed protocol; then the segment is split into clusters. Firstly, 100 vehicles are allocated by uniform distribution to the segment, and constant velocity is assigned to each vehicle. As simulation results, the proposed protocol's output is compared to the CBLTR and AODV-CV regarding PDR, throughput, and E2E delay. Tab. 1 provides a detailed list of parameters for the simulation.

Table 1: Simulation parameters

\begin{tabular}{ll}
\hline Simulation Parameter & Simulation Value \\
\hline Simulation Time & $1000-5000 \mathrm{sec}$ \\
Area & $1000 \times 1000$ \\
Number of Vehicles & $50-100$ \\
Communication Range & 250 units \\
Vehicle Speed Range & $(10-60) \mathrm{kmph}$ \\
Packet Size & 1024 \\
\hline
\end{tabular}

The throughput relation between the proposed protocols, CBCLR, and AODV-CV for 50, 60, 80, and 100 nodes is shown in Figs. 3-6, which shows that throughput increases with the number of nodes increasing. 


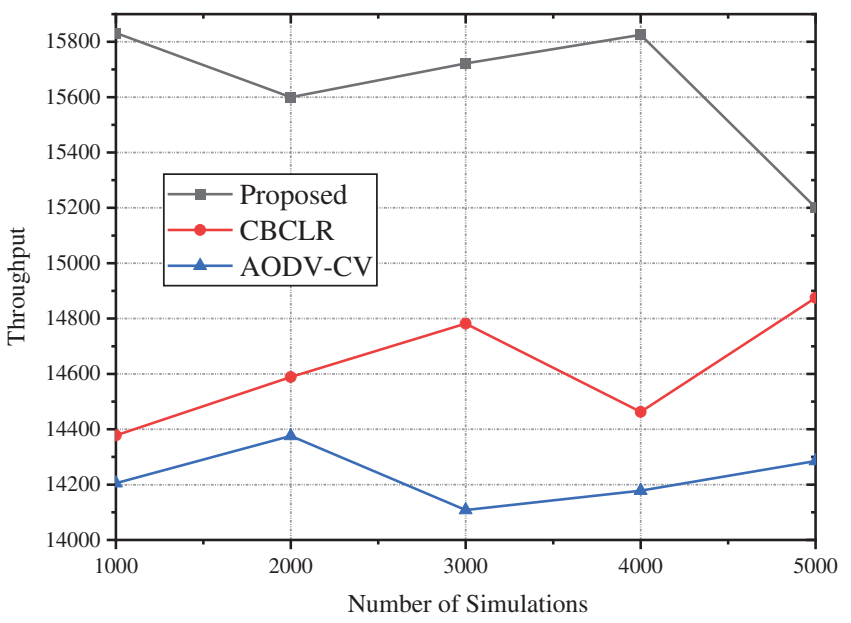

Figure 3: Throughput comparison for 50 nodes

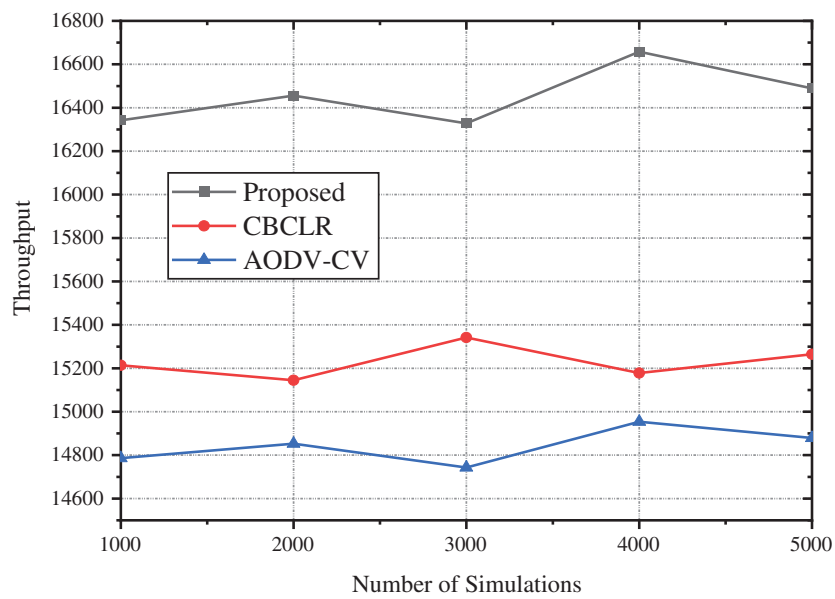

Figure 4: Throughput comparison for 60 nodes

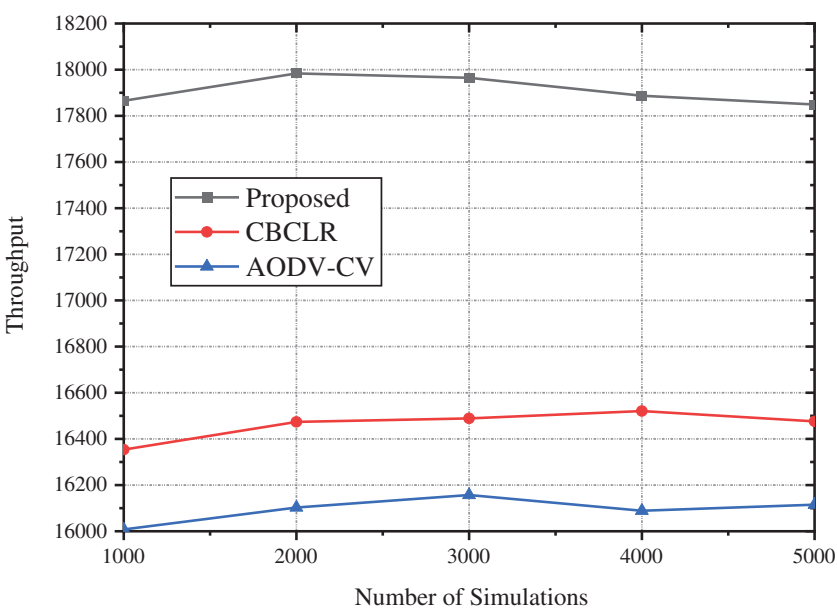

Figure 5: Throughput comparison of 80 nodes 


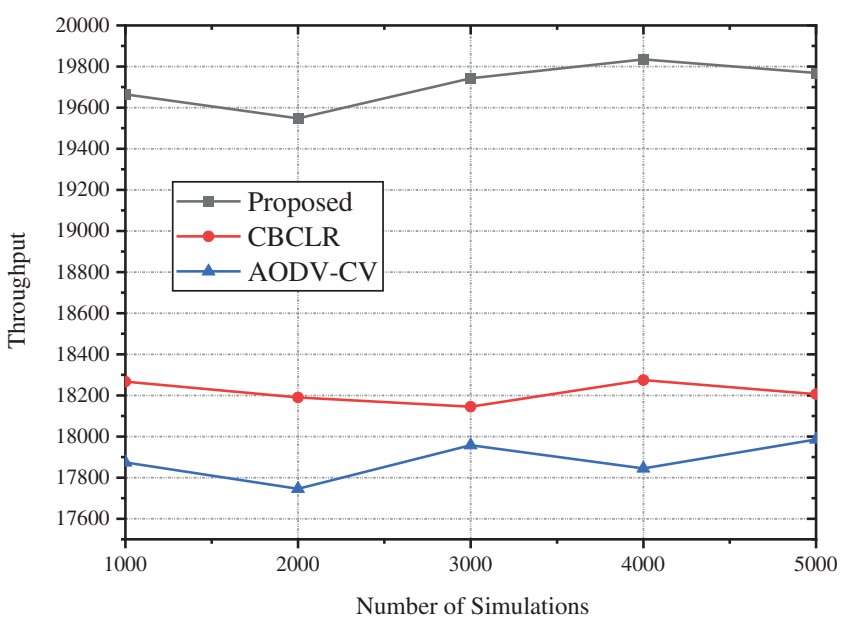

Figure 6: Throughput comparison of 100 nodes

Fig. 7 shows the average comparison of throughput with varying the vehicle nodes for the proposed CBCLR and AODV-CV protocols. The AODV-CV has the least throughput compared to all other protocols as it declined to manage the network changing aspects efficiently compared to CBCLR and proposed protocols. The proposed protocol indicates the throughput improvement compared to CBCLR and AODV-CV because of dynamic clusters creation using K-means and stable $\mathrm{CH}$ election using location, direction, velocity, and POI as the key parameters. The throughput in the proposed protocol is increased by $6.5 \%$ compared to CBCLR protocol and $8.9 \%$ compared to AODV-CV protocol for 50 vehicle nodes in the network.

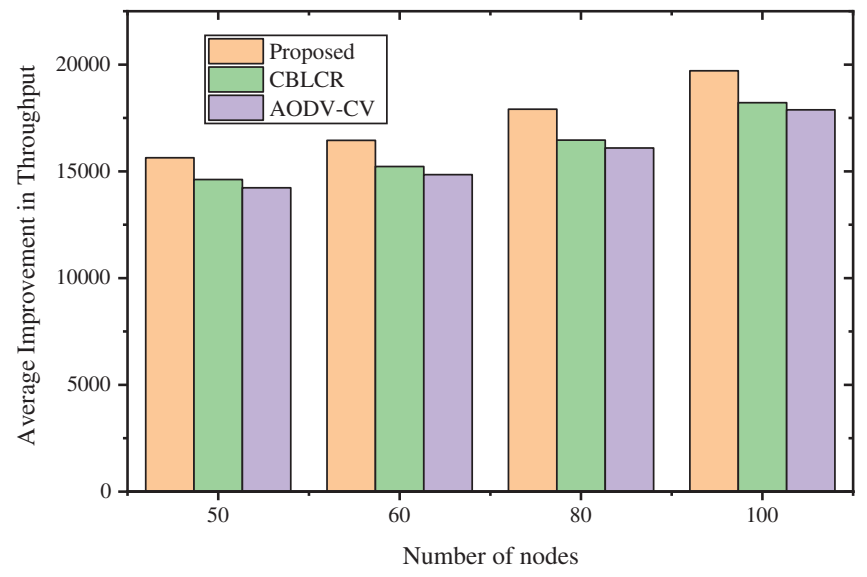

Figure 7: Average improvement in throughput

In Fig. 8, the PDR is computed for the proposed CBCLR and AODV-CV protocols over the various simulations. It is found that PDR remains constant by increasing the number of nodes because PDR is independent of packet injection rate. The PDR in the proposed protocol is improved by $11 \%$ compared to CBCLR protocol and $16.5 \%$ by AODV routing protocol.

In Fig. 9, E2E delay in distribution of packets is computed for the proposed CBCLR and AODV-CV protocols. It is found that the proposed protocol has less delay as compared to CBCLR and AODV-CV. 
This is because the link among the nodes varies as the velocity of vehicles varies. The E2E delay in the proposed protocol is reduced by $46 \%$ compared to CBCLR protocol, $76 \%$ by AODV routing protocol.

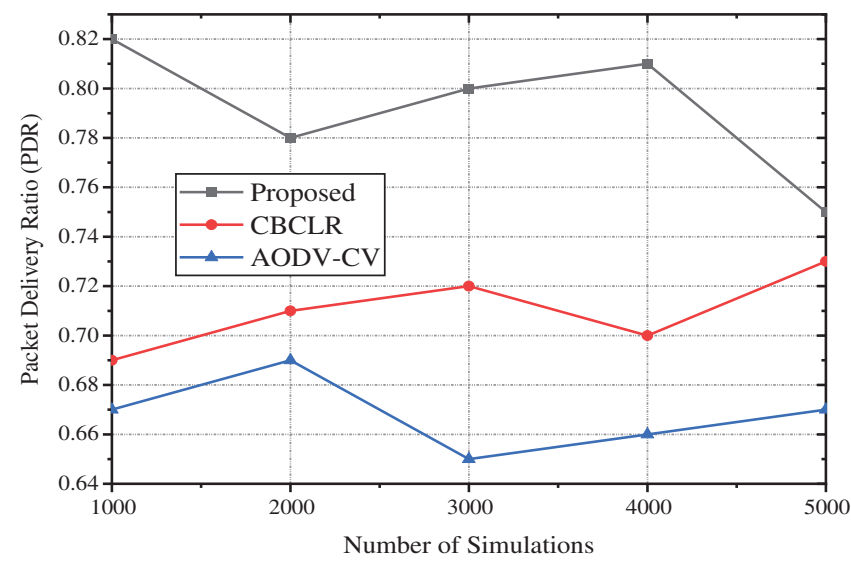

Figure 8: PDR comparison for 50 nodes

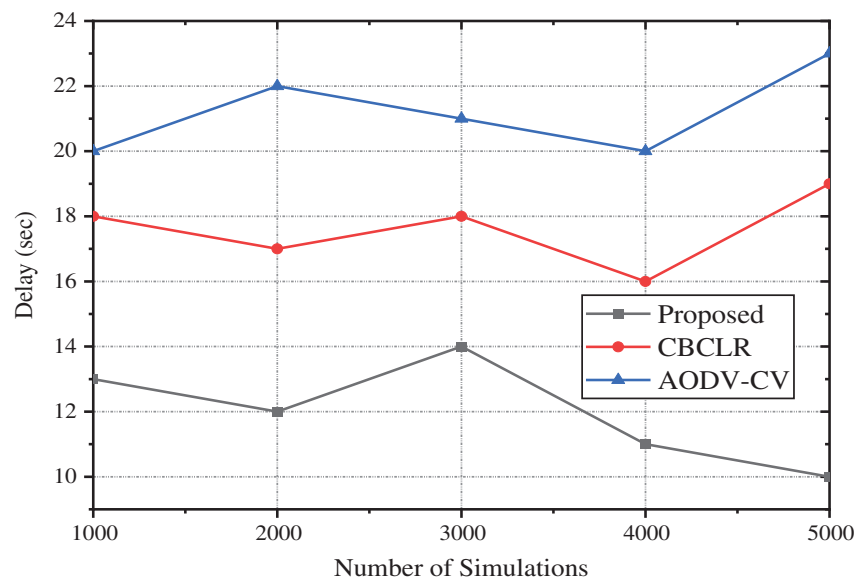

Figure 9: Delay comparison for 50 nodes

\section{Conclusions}

In the paper, a new clustering architecture is proposed that comprises of two algorithms: First, a k-means clustering scheme is suggested, which incorporates regional clustering techniques to minimize overhead and traffic management in VANETs. Second, to choose the next-hop node for inter-clustering routing, a dynamic routing protocol is presented that considers a node's destination, which increases the overall PDR and decreases the E2E latency. The simulation results show that the proposed protocol is more effective as compared to CBCLR and AODV-CV protocols. The comparative analysis indicates that the proposed protocol has up to $6.5 \%$ and $8.9 \%$ more throughput, has up to $11 \%$ and $16.5 \%$ more PDR, and has up to $46 \%$ and $76 \%$ less E2E delay compared to CBCLR and AODV-CV protocols for a varying number of simulations in the network. 
Acknowledgement: The Authors acknowledge Lovely Professional University, Phagwara, Punjab, INDIA for providing state-of-the-art infrastructure to carry out the research.

Funding Statement: The authors received no specific funding for this study.

Conflicts of Interest: The authors declare that they have no conflicts of interest to report regarding the present study.

\section{References}

[1] M. Gerla and J. T. C. Tsai, "Multicluster, mobile, multimedia radio network," Wireless Networks, vol. 1, no. 3, pp. 255-265, 1995.

[2] P. Basu, N. Khan and T. D. Little, "A mobility based metric for clustering in mobile ad hoc networks," in Proc. ICDCSW, Mesa, AZ, USA, pp. 413-418, 2001.

[3] H. Saini and R. Mahapatra, "Implementation and performance analysis of AODV routing protocol in VANETs," International Journal of Emerging Science and Engineering, vol. 2, no. 3, pp. 24-29, 2014.

[4] Z. Y. Rawashdeh and S. M. Mahmud, "A novel algorithm to form stable clusters in vehicular ad hoc networks on highways," EURASIP Journal on Wireless Communications and Networking, vol. 15, no. 1, pp. 1-13, 2012.

[5] A. Çalhan, "A fuzzy logic based clustering strategy for improving vehicular ad-hoc network performance," Indian Academy of Sciences, vol. 40, no. 2, pp. 351-367, 2015.

[6] S. Tayal and M. R. Triphathi, "VANET-challenges in selection of vehicular mobility model," in Proc. 2nd ICACCT, Rohtak, India, pp. 231-235, 2012.

[7] G. Swathi, "A frame work for categorise the innumerable vulnerable nodes in mobile adhoc network," International Journal of Computer Systems Science \& Engineering, vol. 35, no. 5, pp. 335-345, 2020.

[8] S. A. Mohammad and C. W. Michele, "Using traffic flow for cluster formation in vehicular ad-hoc networks," in Proc. IEEE local computer network conf., Denver, CO, USA, pp. 631-636, 2010.

[9] C. Cooper, D. Franklin, M. Ros, F. Safaei and M. Abolhasan, "A comparative survey of VANET clustering techniques," IEEE Communications Surveys \& Tutorials, vol. 19, no. 1, pp. 657-681, 2016.

[10] H. Bello-Salau, A. Aibinu, Z. Wang, A. Onumanyi, E. Onwuka et al., "An optimized routing algorithm for vehicle ad-hoc networks," Engineering Science and Technology, an International Journal, vol. 22, no. 3, pp. 754-766, 2019.

[11] T. Song, W. Xia, T. Song and L. Shen, "A cluster-based directional routing protocol in VANET," in Proc. IEEE 12th int. conf. on communication technology, Nanjing, China, pp. 1172-1175, 2010.

[12] M. Jerbi, S.-M. Senouci, T. Rasheed and Y. Ghamri-Doudane, "Towards efficient geographic routing in urban vehicular networks," IEEE Transactions On Vehicular Technology, vol. 58, no. 9, pp. 5048-5059, 2009.

[13] C. Zhao, C. Li, L. Zhu, H. Lin and J. Li, "A vehicle density and load aware routing protocol for VANETs in city scenarios," in Proc. WCSP, Huangshan, China, pp. 1-6, 2012.

[14] A. Abuashour and M. Kadoch, "Performance improvement of cluster-based routing protocol in VANET," IEEE Access, vol. 5, pp. 15354-15371, 2017.

[15] S. Tabatabaei, "A novel fault tolerance energy-aware clustering method via social spider optimization (SSO) and fuzzy logic and mobile sink in wireless sensor networks (WSNs)," Computer Systems Science and Engineering, vol. 35, no. 6, pp. 477-494, 2020.

[16] A. Louazani, S. M. Senouci and M. A. Bendaoud, "Clustering-based algorithm for connectivity maintenance in vehicular ad-hoc networks," in Proc. ICICS, Reims, France, pp. 34-38, 2014.

[17] A. Malathi and N. Sreenath, "An efficient clustering algorithm for Vanet," International Journal of Applied Engineering Research, vol. 12, no. 9, pp. 2000-2005, 2017.

[18] M. Mohammed Nasr, A. Abdelgader, Z. G. Wang and L. F. Shen, "VANET clustering based routing protocol suitable for deserts," Sensors, vol. 16, no. 4, pp. p.-478, 2016. 
[19] I. Hussain and C. Bingcai, "Cluster formation and cluster head selection approach for vehicle ad-hoc network (VANETs) using K-means and floyd-Warshall technique," International Journal of Advanced Computer Science and Applications, vol. 8, no. 12, pp. 11-15, 2017.

[20] M. Ramalingam and R. Thangarajan, "Mutated k-means algorithm for dynamic clustering to perform effective and intelligent broadcasting in medical surveillance using selective reliable broadcast protocol in VANET," Computer Communications, vol. 150, pp. 563-568, 2020.

[21] A. A. Jamel and B. Akay, "A survey and systematic categorization of parallel k-means and fuzzy-c-means algorithms," International Journal of Computer Systems Science \& Engineering, vol. 34, no. 5, pp. 259-281, 2019.

[22] H. W. Tseng, R. Y. Wu and C. W. Lo, "A stable clustering algorithm using the traffic regularity of buses in urban VANET scenarios,” J Wireless Com Network, vol. 26, pp. 2665-2679, 2020.

[23] X. Ji, H. Yu, G. Fan, H. Sun and L. Chen, "Efficient and reliable cluster-based data transmission for vehicular ad hoc networks," Mobile Information Systems, vol. 2018, pp. 1-15, 2018.

[24] C. J. Joshua and V. Varadarajan, "An optimization framework for routing protocols in VANETs: A multi-objective firefly algorithm approach," Wireless Networks, pp. 1-10, 2019.

[25] R. Sadakale, N. Ramesh and R. Patil, "TAD-HOC routing protocol for efficient VANET and infrastructureoriented communication network," Journal of Engineering, vol. 2020, pp. 1-12, 2020. 